\title{
The Problem of the Twin Paradox Elucidated Based on a Thought Experiment Carried out by Discriminating Between a Classically Stationary Frame and Moving Frame
}

Suto $\mathbf{K}^{*}$

Independent Researcher, 5-24, Oote-Town, Isesaki, Japan

\begin{abstract}
Einstein assumed the "principle of relativity" when constructing the special theory of relativity (STR). He claimed that all inertial frames are equivalent, and that it is impossible to discriminate between inertial frames. However, among the coordinate systems regarded by Einstein as inertial frames, there are some in which light propagates isotropically, and some in which light propagates anisotropically. A method has been found which discriminates between these. This paper discusses the "triplet thought experiment" in which accelerated motion is eliminated from the famous twin paradox thought experiment of the STR. Here the inertial frames regarded as equivalent by the STR are identified as "classically stationary frames $S_{c}$ " and "classically moving frames $\left(S_{\mathrm{cl}}^{\prime}\right)$ " First, an observer $M$ in frame performs the triplet thought experiment, and it is confirmed that the delay in time which elapses in the moving frame agrees with the predictions of the STR. Next, an observer in rocket A $\left(S_{\mathrm{cl}}^{\prime}\right)$ performs the triplet thought experiment, and observes the same time delay. Before starting movement at constant velocity, rocket $A$ experiences accelerated motion. The coordinate system of rocket $A$ cannot be regarded physically as a stationary frame. Even so, observer A observes the delay predicted by the STR. If it can be accepted that frames $S_{C}$ are included in inertial frames, then the problem of the twin paradox will no longer be a paradox.
\end{abstract}

Keywords: Twin paradox; Triplet thought experiment; Classically stationary frame; Relativistically stationary frame; Classically moving frame; Minkowski diagram; Velocity vector

\section{Introduction}

Among the hypothetical paradoxes generated by the special theory of relativity (STR), the twin paradox (or clock paradox) is the most famous.

Suppose two clocks have been synchronized to the current time, and mark time at the same rhythm. Assume that one clock (the first clock) remains stationary in a certain inertial frame and the other clock (the second clock) is carried away along an arbitrary path, eventually returning to the departure point. The STR predicts that, at this time, the second clock will be delayed compared to the first clock [1].

To use a modern example, if the older of two twin astronauts returns from a trip through space, he will find that he is younger than his younger brother who remained on earth. This problem has been vigorously debated in the past, and today the issue is thought to be settled [2].

The tradition view put forward to avoid the paradox is as follows:

"The coordinate frame of the second clock moving with respect to the inertial frame undergoes accelerated motion, and thus an asymmetry exists between the two coordinate frames. The side which has moved is clearly the second clock, and thus it is natural for the second clock to be delayed."

A coordinate system which has attained movement at constant velocity through accelerated motion cannot be regarded physically as a stationary frame.

However, this paper presents a thought experiment performed in a coordinate system which cannot be regarded as this stationary frame, and in this experiment a delay of time is observed which agrees with the prediction of the STR. However, in order to avoid discussion of the accelerated motion treated in the twin paradox thought experiment, here the author considers the "triplet thought experiment."

The discussion in this case is simplified if one of the multiple coordinate systems treated in the thought experiment is a classically stationary frame in which light propagates isotropically.

Thus, this paper first presents the fact that there is a method for identifying inertial frames as "classically stationary frames $S_{\mathrm{cl}}$ " and "classically moving frames $S_{\mathrm{cl}}^{\prime}$ " in which light propagates anisotropically. (However, the STR does not recognize these categories due to the "principle of relativity").

Also, the "triplet thought experiment" is performed by introducing an inertial frame experimentally confirmed to be frame $S_{\mathrm{cl}}$.

\section{Time That Is Actually Adjusted in Synchronization of the Two Clocks}

Let there be a given stationary rigid rod of length $L$ as measured by a ruler which is stationary, and assume that the rod is placed along the stationary frame's $x$-axis.

Assume that clocks A and B of the same type are set up at points $\mathrm{A}$ and $\mathrm{B}$ on the rear and front end of this rod. Here clock $\mathrm{A}$ will be abbreviated as $\mathrm{C}_{\mathrm{A}}$, and clock $\mathrm{B}$ as $\mathrm{C}_{\mathrm{B}}$.

Suppose a ray of light is emitted in the direction of $\mathrm{B}$ from $\mathrm{A}$ at time $t_{\mathrm{A}}$ of $\mathrm{C}_{\mathrm{A}}$, reaches and is reflected at $\mathrm{B}$ at time $t_{\mathrm{B}}$ of $\mathrm{C}_{\mathrm{B}}$, and then

*Corresponding author: Suto K, Independent Researcher, 5-24, OoteTown, Isesaki, 372-0048, Japan, Tel: 011+81-270-23-9980; E-mail: koshun_suto129@mbr.nifty.com

Received May 31, 2018; Accepted July 26, 2018; Published August 02, 2018

Citation: Suto K (2018) The Problem of the Twin Paradox Elucidated Based on a Thought Experiment Carried out by Discriminating Between a Classically Stationary Frame and Moving Frame. J Phys Math 9: 278. doi: 10.4172/2090-0902.1000278

Copyright: () 2018 Suto K. This is an open-access article distributed under the terms of the Creative Commons Attribution License, which permits unrestricted use, distribution, and reproduction in any medium, provided the original author and source are credited. 
returns to $\mathrm{A}$ at time $t_{\mathrm{A}^{\prime}}$ of $\mathrm{C}_{\mathrm{A}}$. Einstein determined that if the following relationships hold between these times, then the two clocks represent the same time by definition [3].

$$
t_{\mathrm{B}}-t_{\mathrm{A}}=t_{\mathrm{A}^{\prime}}-t_{\mathrm{B}} \text {. }
$$

If the relationship in eqn. (1) does not hold for the times of $C_{A}$ and $\mathrm{C}_{\mathrm{B}}$, then it is necessary to adjust the time of $\mathrm{C}_{\mathrm{B}}$ so that the relationship in eqn. (1) holds. (Actually, either clock can be adjusted).

Now, consider the case where two rods are placed in frame $S_{\mathrm{cl}}$ (The two rods will be distinguished as rod I and rod II) (Figure 1).

On $\operatorname{rod} \mathrm{I}$, clock $\mathrm{A}$ will be indicated as $\mathrm{C}_{\mathrm{IA}}$ and clock $\mathrm{B}$ will be indicated as $\mathrm{C}_{\mathrm{IB}}$ ( $\mathrm{In}_{\mathrm{IA}}$, I indicates rod $\mathrm{I}$, and $\mathrm{A}$ indicates clock $\mathrm{A}$. The same holds for $\mathrm{C}_{\mathrm{IB}}$ ). The clocks at both ends of rod II will be indicated as $\mathrm{C}_{\mathrm{IIA}}$ and $\mathrm{C}_{\mathrm{IIB}}$.

It is assumed that the times of $\mathrm{C}_{\mathrm{IA}}$ and $\mathrm{C}_{\mathrm{IB}}$, as well as $\mathrm{C}_{\mathrm{IIA}}$ and $\mathrm{C}_{\mathrm{IIB}}$ are synchronized when the clocks are at rest. (Here, the adjustment time is not important. It is sufficient to just synchronize the times of the two clocks.)

Once their times have been adjusted, $\mathrm{C}_{\mathrm{IB}}$ will be indicated as $\mathrm{C}_{\mathrm{IB1}}$, and $\mathrm{C}_{\mathrm{IIB}}$ will be indicated as $\mathrm{C}_{\mathrm{IIB1}}$. The 1 in ${ }_{\mathrm{B} 1}$ refers to the first time adjustment.

Here, the times are synchronized when the two clocks are stationary because the author wishes to carry the discussion up to the time adjustment when performing synchronization later.

Also, if the stationary frame is a frame $S_{\mathrm{cl}}$, then $\mathrm{C}_{\mathrm{IA}}$ and $\mathrm{C}_{\mathrm{IB1}}$, and $\mathrm{C}_{\mathrm{IIA}}$ and $\mathrm{C}_{\mathrm{IIB1}}$, match in an absolute sense.

Next, consider the case when rod I and rod II begin to move at a constant velocity, in the positive direction of the $x$-axis of the stationary frame (Velocity of rod I is assumed to be $v$, and velocity of rod II to be $u$ ). It is assumed here that $v<u$ (Figure 2).

Here, when clock $B$ on rod $I$ is adjusted, the indication $C_{I B 1}$ is changed to $\mathrm{C}_{\mathrm{IB2}}$. Also, when clock $\mathrm{B}$ on rod II is adjusted, the indication $\mathrm{C}_{\mathrm{IIB} 1}$ is changed to $\mathrm{C}_{\mathrm{IIB} 2}$. The 2 in ${ }_{\mathrm{B} 2}$ refers to the second time adjustment.

Adjustment time $\Delta t_{1}$ of clock B in the coordinate system $S_{\mathrm{I}}^{\prime}$ of rod I and adjustment time $\Delta t_{2}$ of clock B in coordinate system $S_{\text {II }}^{\prime}$ of rod II predicted by an observer in frame $S_{\mathrm{cl}}$.

\begin{tabular}{llr}
$\mathrm{C}_{\mathrm{IIA}}$ & $\mathrm{C}_{\mathrm{IIBI}}$ \\
\hline (1) & Rod II & (1) \\
\hline A & & B \\
\hline $\mathrm{C}_{\mathrm{IA}}$ & & $\mathrm{C}_{\mathrm{IBI}}$ \\
\hline
\end{tabular}

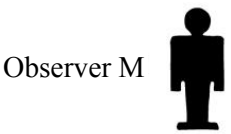

Classically stationary frame

Figure 1: Two rods with length $L$ are placed parallel to the $x$-axis of a "classically stationary frame $S_{\mathrm{cl}}$ " At this time, the clocks at both ends of the two rods are synchronized.

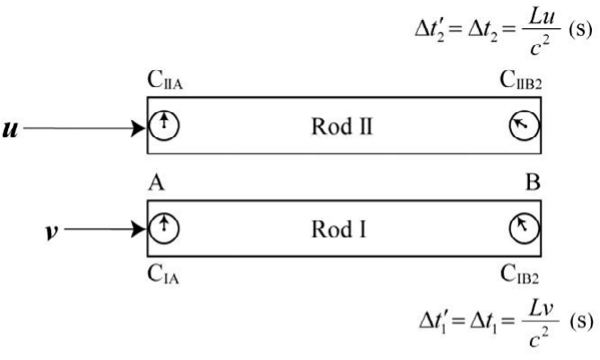

\section{Observer M

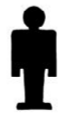 \\ Classically stationary frame}

Figure 2: Time adjustment of $C_{1 B 1}$ moving at constant velocity $v$ relative to a "classically stationary frame" and time adjustment of $\mathrm{C}_{1 \mathrm{~B} 1}$ moving in the same way at constant velocity $u$. By making this time adjustment, the coordinate systems of rod I and rod II can maintain their status as "relativistically stationary frames." In this figure, $\Delta t_{1}^{\prime}$ and $\Delta t_{2}^{\prime}$ are times for adjustment by the observer of rod $\mathrm{I}$ and rod $\mathrm{II}$, and $\Delta t_{1}$ and $\Delta t_{2}$ are adjustment times predicted by the observer $\mathrm{M}$.

Let us measure the time of an event which occurs in another inertial frame by using the clock in frame $S_{\mathrm{cc}}$. Here, it is assumed that the times $t_{\mathrm{A}}^{\prime}, t_{\mathrm{B}}^{\prime}, t_{\mathrm{A}^{\prime}}^{\prime}$ in frame ' correspond to the times $t_{\mathrm{A}}, t_{\mathrm{B}}, t_{\mathrm{A}^{\prime}}$ measured from frame $S_{\mathrm{cl}}$.

When the time needed for light emitted from $\mathrm{A}$ in frame $S_{\mathrm{I}}^{\prime}$ to B is measured with the clock in stationary frame, the result is $\left(t_{\mathrm{B}}-t_{\mathrm{A}}\right)$.

According to the STR, when viewed from stationary frame, the rod I contracts by $1 / \gamma$ times in the direction of motion. In addition, when the light speed emitted from frame $S_{\mathrm{I}}^{\prime}$ is seen from stationary frame, it is always constant regardless of the velocity of the light source, and thus $\left(t_{\mathrm{B}}-t_{\mathrm{A}}\right)$ is given by the following equation.

$$
t_{\mathrm{B}}-t_{\mathrm{A}}=\frac{L}{\gamma(c-v)}(\mathrm{s}), \gamma=\left(1-\frac{v^{2}}{c^{2}}\right)^{-1 / 2} \text {. }
$$

If the time needed for light to return from $\mathrm{B}$ to $\mathrm{A}$ is measured with the clock in stationary frame, and is taken to be $\left(t_{\mathrm{A}^{\prime}}-t_{\mathrm{B}}\right)$ then:

$$
t_{\mathrm{A}^{\prime}}-t_{\mathrm{B}}=\frac{L}{\gamma(c+v)}(\mathrm{s}) \text {. }
$$

However, the denominator on the right side of eqns. (2) and (3) does not mean that the light speed varies depend on the velocity of the light source [4].

According to the STR, the relationship between the time $\left(t_{\mathrm{B}}^{\prime}-t_{\mathrm{A}}^{\prime}\right)$ that elapses in frame $S_{\mathrm{I}}^{\prime}$ and the time $\left(t_{\mathrm{B}}-t_{\mathrm{A}}\right)$ that elapses on a clock in frame $S_{\mathrm{cl}}$ is as follows.

$$
t_{\mathrm{B}}^{\prime}-t_{\mathrm{A}}^{\prime}=\frac{1}{\gamma}\left(t_{\mathrm{B}}-t_{\mathrm{A}}\right)
$$

If the right side of eqn. (2) is substituted for $\left(t_{\mathrm{B}}-t_{\mathrm{A}}\right)$ in eqn. (4),

$$
\begin{aligned}
& t_{\mathrm{B}}^{\prime}-t_{\mathrm{A}}^{\prime}=\frac{L}{\gamma^{2}(c-v)} \\
& =\frac{L(c+v)}{c^{2}} \text { (s). }
\end{aligned}
$$

If, in the same way, the time elapsed on a clock in frame $S_{\mathrm{I}}^{\prime}$ while light returns from $\mathrm{B}$ to $\mathrm{A}\left(t_{\mathrm{A}^{\prime}}^{\prime}-t_{\mathrm{B}}^{\prime}\right)$ is: 


$$
t_{\mathrm{A}^{\prime}}^{\prime}-t_{\mathrm{B}}^{\prime}=\frac{L(c-v)}{c^{2}}(\mathrm{~s})
$$

If we set $t_{\mathrm{A}}^{\prime}=0$ to simplify the formula, then the following value is obtained from eqns. (6) and (7).

$$
\begin{aligned}
& \frac{1}{2} t_{\mathrm{A}^{\prime}}^{\prime}=\frac{1}{2}\left[\left(t_{\mathrm{B}}^{\prime}-t_{\mathrm{A}}^{\prime}\right)+\left(t_{\mathrm{A}^{\prime}}^{\prime}-t_{\mathrm{B}}^{\prime}\right)\right] \\
& =\frac{1}{2}\left[\frac{L(c+v)}{c^{2}}+\frac{L(c-v)}{c^{2}}\right] \\
& =\frac{L}{c} \text { (s). }
\end{aligned}
$$

When light travels from A to B in frame $S_{I}^{\prime}$, an observer in stationary frame predicts that $L(c+v) / c^{2}$ (s) have passed on the clock in frame $S_{\mathrm{I}}^{\prime}$. However, when this light which left A at $t_{\mathrm{A}}^{\prime}=0$ reaches B, by definition, the time shown on clock B must be $L / c$ (s).

However, since $L(c+v) / c^{2}>L / c$, the time on clock B must be later than the time on clock $A$ to resolve this discrepancy. Thus, if the time adjustment to actually make the time on clock B later is $\Delta t_{1}^{\prime}$, it should be possible to take the difference between the two as this time.

$$
\begin{aligned}
& \text { Namely, } \\
& \Delta t_{1}^{\prime}=\left(t_{\mathrm{B}}^{\prime}-t_{\mathrm{A}}^{\prime}\right)-\frac{1}{2} t_{\mathrm{A}^{\prime}}^{\prime} \\
& =\frac{L(c+v)}{c^{2}}-\frac{L}{c} \\
& =\frac{L v}{c^{2}}(\mathrm{~s}) .
\end{aligned}
$$

$\Delta t_{1}^{\prime}$ matches with the adjustment time $\Delta t$ predicted by an observer in the stationary frame.

If an observer in frame $S_{\mathrm{I}}^{\prime}$ delays the time on clock B by $L v / c^{2}(\mathrm{~s})$, then the relationship in eqn. (1) will hold in this coordinate system.

Now we synchronize the time of clocks on both ends of rod II. In this case too, there is a match between the adjustment time $\Delta t_{2}$ predicted by an observer in the stationary frame and the adjustment time $\Delta t_{2}^{\prime}$ actually performed by an observer of rod II. That is,

$$
\Delta t_{2}=\Delta t_{2}^{\prime}=\frac{L u}{c^{2}}(\mathrm{~s})
$$

In eqn. (14), the $v$ in eqn. (13) is replaced with $u$.

In this case, time adjustment (14) of clock B of rod II is performed by dividing into 2 adjustments.

To begin, the first time adjustment (13) is performed when the velocity of rod II is $v$. Next, rod II is accelerated and when the constant velocity $u$ is attained, the second time adjustment is performed.

If here the adjustment time of clock $\mathrm{B}$, predicted by the observer in frame $S_{\mathrm{cl}}$, is assumed to be $\Delta t_{3}$,

$\Delta t_{3}=\Delta t_{2}-\Delta t_{1}$

$\Delta t_{3}$ is as follows, due to eqns. (14) and (13).

$\Delta t_{3}=\frac{L(u-v)}{c^{2}}=\Delta t_{3}^{\prime}(\mathrm{s})$.

This is equal to the adjustment time $\Delta t_{3}^{\prime}$ actually performed by the observer of rod II.
Incidentally, the velocity addition law in the STR is given by the following formula [5].

$$
u=\frac{v+w}{1+\frac{v w}{c^{2}}} .
$$

This indicates that when the relative velocity of frame $S_{\mathrm{cl}}$ and rod II is $u$, the relative velocity of rod I and rod II is $w$.

Now, observer I predicts the adjustment time of clock B of rod II moving at constant velocity $w$ relative to rod I (Figure 3 ).

The observer of rod I applying the "principle of relativity" regards his own coordinate frame as a stationary frame. Thus, this observer predicts that the adjustment time $\Delta t_{4}$ of clock B on rod II will be as follows.

$$
\Delta t_{4}=\frac{L w}{c^{2}}(\mathrm{~s})
$$

In this case, there is no match between the adjustment time $\Delta t_{4}$ of clock B on rod II predicted by the observer of rod I, and the time $\Delta t_{3}^{\prime}$ of actual adjustment by the observer of rod II. That is,

$$
\Delta t_{4} \neq \Delta t_{3}^{\prime} .
$$

A velocity vector is attached to the coordinate system of rod I, which is regarded as a stationary frame from the standpoint of the "principle of relativity" [4-7]. Since this sort of inertial frame exists in the natural world, the "principle of relativity" that regards all inertial frames as equivalent, cannot be regarded as a true principle [8-10].

The results of obtained thus far are summarized in the following Table 1.

\section{Also, this velocity vector is defined in the following.}

According to the quantum electrodynamics, a vacuum which transmits electrical force is thought to be filled with opposing pairs of virtual particles and antiparticles. The vacuum can transmit light as a wave. Therefore, let us tentatively assume that these virtual particles are the modern day ether. Also, according to the "uncertainty principle," these virtual particles are constantly fluctuating and not at rest, even when in the lowest energy state.

Here, it is assumed that a vacuum exists even at the deep layer of a single arbitrary point in the space of an inertial frame. Next, vectors

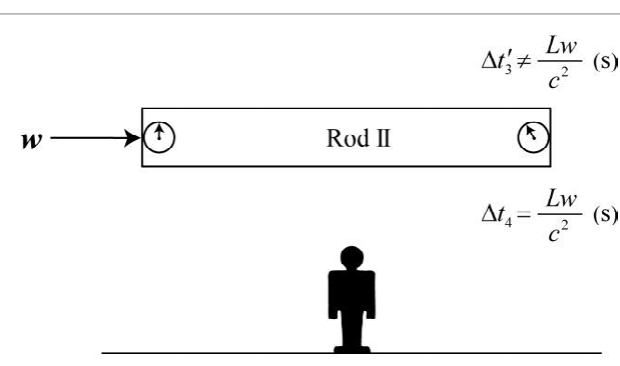

Observer on Rod I

Classically moving frame

Figure 3: The case when rod II in Figure 2 is viewed by the observer of rod I. In this case, the observer of rod I believes that his own coordinate system is a stationary frame, and thus he believes that the time adjustment of $\Delta t_{4}$ $\mathrm{C}_{\text {III }}$ is $L w / c^{2}$ (s). This time does not match the time of $\Delta t_{3}^{\prime}$ adjustment by the observer of rod II. The cause of the mismatch between $\Delta t$ and $\Delta t^{\prime}$ is the velocity vector attached to the coordinate system of rod I (Figure 2) regarded as a stationary frame from the perspective of the STR. 
Citation: Suto K (2018) The Problem of the Twin Paradox Elucidated Based on a Thought Experiment Carried out by Discriminating Between a Classically Stationary Frame and Moving Frame. J Phys Math 9: 278. doi: 10.4172/2090-0902.1000278

Page 4 of 8

\begin{tabular}{|c|c|c|}
\hline Inertial frames (Relativistically stationary frames) & Classically stationary frames & Classically moving frames \\
\hline Propagation of light in stationary frame & A priori isotropic propagation & Anisotropic propagation \\
\hline $\begin{array}{c}\text { Adjustment time of clock in moving frame predicted by observer M, } \\
\text { and time of actual adjustment by observer in moving frame }\end{array}$ & Does not matches \\
\hline Velocity vector attached to stationary frame & Does not exist & Exists \\
\hline
\end{tabular}

Table 1: Discrimination of inertial frames that cannot be discriminated in the STR. Einstein assumed the "principle of relativity," and claimed that all inertial frames are equivalent, but there is a thought experiment that overturns that claim.

are used to indicate the velocities at a certain time of the countless virtual particles which exist at that point in the vacuum, and then those vectors are combined into a single vector. (If there is a problem here with the expression "which exist at the point," it can be changed to the more ambiguous expression "which exist in the neighborhood of that point"). This combined vector is taken to be the velocity vector at that point.

Next, a vector is used to indicate the relative velocity between the combined vector and the inertial frame.

If the relative velocity is zero, this inertial frame is determined to be "classically stationary frame."

Conversely, if the relative velocity is not zero, this inertial frame is determined to be "classically moving frame." However, what determines the direction of this vector is convention.

In this paper, the author feels it is best treat this vector as having a starting point in the vacuum and an end point in the inertial frame of physical space.

In this case, the point in the vacuum plays the role of a stationary frame. Also, the $v_{x}$ is the component in the $x$-axis direction of the velocity vector attached to the inertial frame regarded as a problem here.

\section{Three Kinds of “Triplet Thought Experiment"}

\section{A. Triplet Thought Experiment 1 Performed by Observer M}

Rocket $\mathrm{A}$ is moving at a constant velocity $0.6 \mathrm{c}$ in the $x$-axis direction of the coordinate system $\mathrm{M}\left(S_{\mathrm{M}}\right)$ (Figure $4 \mathrm{a}$ ).

In preparation for the thought experiment to be conducted, it is assumed that it has been confirmed through an experiment beforehand that frame $S_{\mathrm{M}}$ is a classically stationary frame $S_{\mathrm{cl}}$ (The coordinate system of rocket A, classically moving frame $S_{\mathrm{cl}}^{\prime}$, is described as frame $S_{\mathrm{A}}^{\prime}$ ).

There is an observer $\mathrm{M}$ at the origin $\mathrm{O}$ of the $x$-axis of frame $S_{\mathrm{cl}}$ and $\mathrm{M}$ has a stop watch $\mathrm{W}$. In addition, there is an observer $\mathrm{A}$ at the origin $\mathrm{O}_{\mathrm{A}}^{\prime}$ of the $x_{\mathrm{A}}^{\prime}$-axis of frame $S_{\mathrm{A}}^{\prime}$, and $\mathrm{A}$ has a stopwatch $\mathrm{W}_{\mathrm{A}}$.

Now, when rocket $A$ passes in front of observer $M$, observer $M$ starts $\mathrm{W}$, and observer A starts $\mathrm{W}_{\mathrm{A}}$. Then, when 1(s) has elapsed on $\mathrm{W}$, rocket $\mathrm{A}$ passes by rocket $\mathrm{B}$ that has approached from the forward direction (Figure $4 \mathrm{~b}$ ).

At this time, observer $\mathrm{A}$ stops $\mathrm{W}_{\mathrm{A}}$, and observer $\mathrm{B}$ on rocket $\mathrm{B}$ starts stop watch $\mathrm{W}_{\mathrm{B}}$. (However, it is assumed that the velocity of rocket $\mathrm{B}$ measured by an observer in frame $S_{\mathrm{cl}}$ is $-0.6 \mathrm{c}$.)

According to the STR, an observer in frame $S_{\mathrm{cl}}$ finds the following relationship between the time $t$ which elapses on $\mathrm{W}$ and the time $t^{\prime}$ which elapses on $\mathrm{W}_{\mathrm{A}}$.

$$
t_{\mathrm{A}}^{\prime}=\frac{t}{\gamma}=t\left(1-\frac{v^{2}}{c^{2}}\right)^{1 / 2} \text {. }
$$

Here, when $1(\mathrm{~s})$ is substituted for $t$,
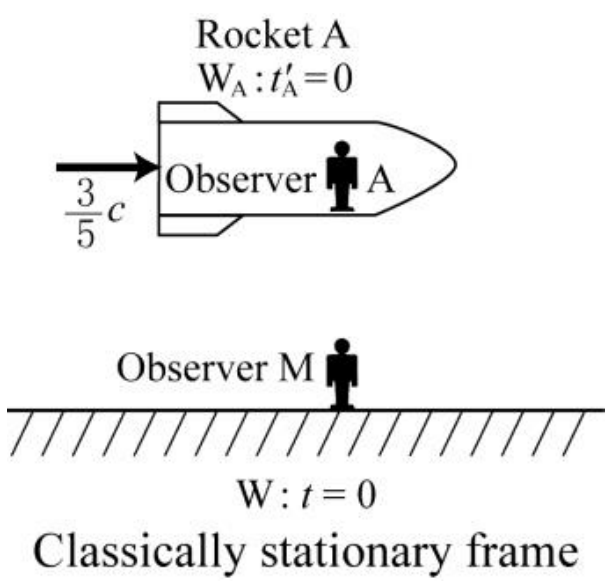

Figure 4a: When the observer on rocket $A$ passes in front of observer $M$, the two observers start their own stop watches.

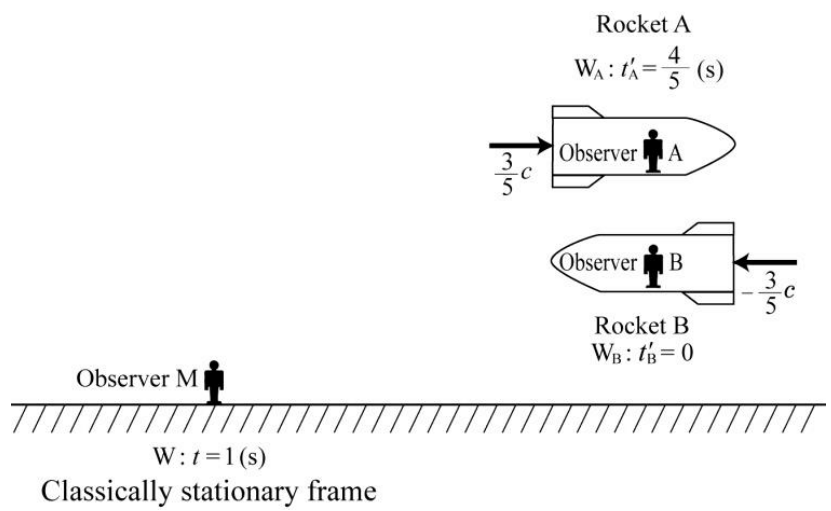

Figure 4b: Instant when rocket $A$ and rocket $B$ pass by each other. At this time, observer $A$ stops $W_{A}$, and observer $B$ on rocket $B$ starts stop watch $W_{B}$.

$t_{\mathrm{A}}^{\prime}=\frac{4}{5}(\mathrm{~s})$

Next, when the observer in rocket B, who continues to move at constant velocity, passes in front of observer $M$, the two observers stop their stop watches (Figure 4c).

If the time elapsed on $\mathrm{W}_{\mathrm{B}}$ at this time is taken to be $t_{\mathrm{B}}^{\prime}$, then since $t_{\mathrm{B}}^{\prime}$ is equal to eqn. (21). That is,

$t_{\mathrm{B}}^{\prime}=\frac{4}{5}(\mathrm{~s})$.

On the other hand, the time elapsed on W in frame $S_{\mathrm{cl}}$ is 2 (s). According to the STR, during the interval where 2 (s) elapses on $\mathrm{W}$, both the time $t_{\mathrm{A}}$ which elapses on $\mathrm{W}_{\mathrm{A}}$ and the time $t_{\mathrm{B}}^{\prime}$ which elapses on $\mathrm{W}_{\mathrm{B}}$ are both $0.8(\mathrm{~s})$. Therefore, an observer in $S_{\mathrm{cl}}$ derives the following relationship from $t, t_{\mathrm{A}}^{\prime}$ and $t_{\mathrm{B}}^{\prime}$. 


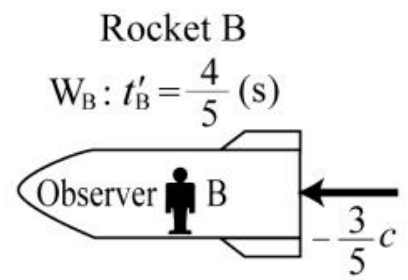

\section{Observer $\mathrm{M}$ i \\ $\mathrm{W}: t=2(\mathrm{~s})$ \\ Classically stationary frame}

Figure 4c: When the observer on rocket $B$ passes in front of observer $M$, the two observers stop their stop watches.

$$
\begin{aligned}
& t=2(\mathrm{~s}), t_{\mathrm{A}}^{\prime}=\frac{4}{5}(\mathrm{~s}), t_{\mathrm{B}}^{\prime}=\frac{4}{5}(\mathrm{~s}) . \\
& t:\left(t_{\mathrm{A}}^{\prime}+t_{\mathrm{B}}^{\prime}\right)=1: \frac{4}{5} .
\end{aligned}
$$

The time $\left(t_{\mathrm{A}}^{\prime}+t_{\mathrm{B}}^{\prime}\right)$ which passes on rockets $\mathrm{A}$ and $\mathrm{B}$ moving at constant velocity is delayed compared to the time $t$ which elapses in $S_{\mathrm{cl}}$. Thought experiment 1 is an experiment in which accelerated movement has been removed from the famous twin paradox, and is called the "triplet thought experiment." (In this case, the triplets correspond to $\mathrm{W}, \mathrm{W}_{\mathrm{A}}$ and $\mathrm{W}_{\mathrm{B}}$ ).

Here, this thought experiment is explained using Minkowski diagram 1 (Figure 5).

The following explanation in this section is an excerpt from another paper [11].

Point $\mathrm{O}$ indicates both origins: $x=0, t=0$ and $x_{\mathrm{A}}^{\prime}=0, t_{\mathrm{A}}^{\prime}=0$. The point event $\mathrm{M}_{0}$ of stop watch $\mathrm{W}$ of frame $S_{\mathrm{cl}}$ and the point event $\mathrm{A}_{0}$ of stop watch $\mathrm{W}_{\mathrm{A}}$ are at the origin $\mathrm{O}$. (Here, the subscripts " 0 " of the point events $\mathrm{M}_{0}$ and $\mathrm{A}_{0}$ mean, respectively, $t=0$ and $t_{\mathrm{A}}^{\prime}=0$ ).

The $x$-axis indicates the $x$-axis of the inertial frame $S_{\mathrm{cl}}$ when $t=0$. In addition, the $x_{\mathrm{A}}^{\prime}$-axis indicates the $x_{\mathrm{A}}^{\prime}$-axis of the inertial frame $S_{\mathrm{A}}^{\prime}$ when $t_{\mathrm{A}}^{\prime}=0$. (However, the $x_{\mathrm{B}}^{\prime}$-axis is omitted for brevity),

The $c t$-axis is the path for $x=0$. Put another way, it is the world line of stop watch $\mathrm{W}$. The $c t_{\mathrm{A}}^{\prime}$-axis is the world line of stop watch $\mathrm{W}_{\mathrm{A}}$.

In addition, the straight line extending at a $45^{\circ}$ angle from the origin $\mathrm{O}$ indicates the light signal emitted from the two light sources at the instant that $\mathrm{O}$ and $\mathrm{O}_{\mathrm{A}}^{\prime}$ pass by each other.

$\mathrm{OE}$ is the distance over which the light signal emitted from $\mathrm{O}$ propagates in the $x$-axis direction while 1(s) elapses on the stop watch W in frame $S_{\mathrm{cl}}$.

$\mathrm{OE}^{\prime}$ is the distance over which the light signal emitted from $\mathrm{O}_{\mathrm{A}}^{\prime}$ propagates in the $x_{\mathrm{A}}^{\prime}$-axis direction while $1(\mathrm{~s})$ elapses on the stop watch $\mathrm{W}_{\mathrm{A}}$ in frame $S_{\mathrm{A}}^{\prime}$.

Oe is the value when an observer in frame $S_{\mathrm{cl}}$ measures the distance $\mathrm{OE}^{\prime}$, and $\mathrm{Oe}^{\prime}$ is the value when the distance $\mathrm{OE}$ is measured by an observer in frame $S_{\mathrm{A}}^{\prime}$. However, Ee' is parallel to the $c t$-axis, and $\mathrm{eE}^{\prime}$ is

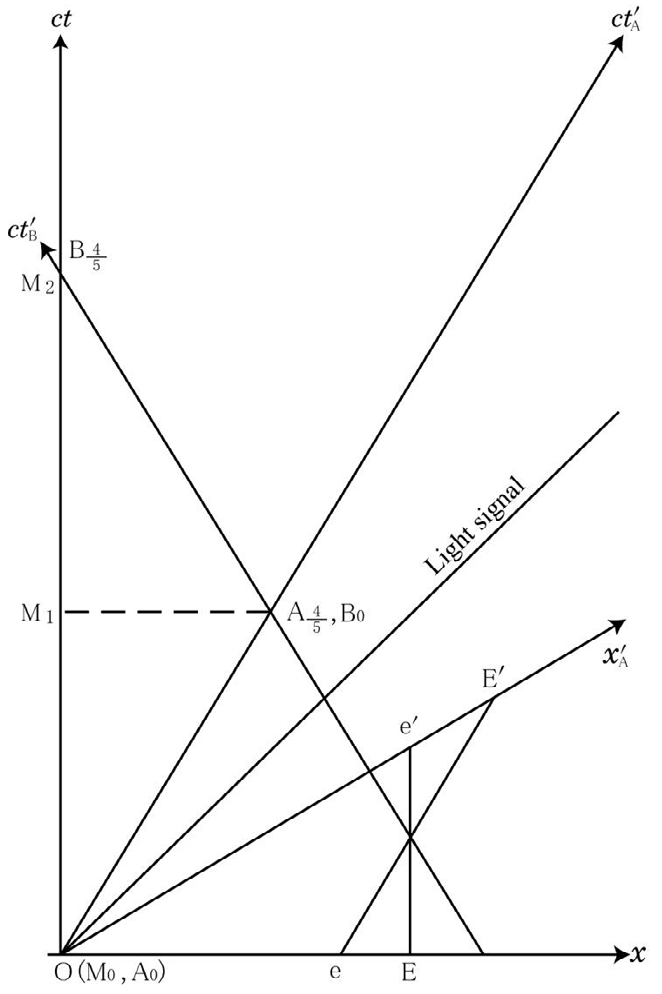

Figure 5: Minkowski diagram 1: This diagram corresponds to thought experiment 1. World lines of stop watches $W_{A}\left(A_{0} A_{4 / 5}\right), W_{B}\left(B_{0} B_{4 / 5}\right)$ and $\mathrm{W}\left(\mathrm{M}_{0} \mathrm{M}_{1} \mathrm{M}_{2}\right)$.

parallel to the $c t_{\mathrm{A}}^{\prime}$-axis. Therefore, the relationship between $\mathrm{OE}, \mathrm{OE}^{\prime}$, Oe and $\mathrm{Oe}^{\prime}$ is as follows.

$$
\frac{\mathrm{Oe}}{\mathrm{OE}^{\prime}}=\frac{\mathrm{Oe}^{\prime}}{\mathrm{OE}}=\frac{1}{\gamma} \text {. }
$$

If a point is plotted on the $c t$-axis at a distance equal to $\mathrm{OE}$ from $\mathrm{O}$, that is the point event $\mathrm{M}_{1}$ of W when $t=1$ (s). Also, if a point is plotted on the $c t_{\mathrm{A}}^{\prime}$-axis at a distance equal to $\mathrm{OE}^{\prime}$ from $\mathrm{O}$, that is the point event $\mathrm{A}_{1}$ of $\mathrm{W}_{\mathrm{A}}$ when $t_{\mathrm{A}}^{\prime}=1(\mathrm{~s})$.

Now, how should we find the relationship between the times which elapse in the stationary frame and in the coordinate system of rocket A?

To find that, it is enough to compare the times when the straight line parallel to the $x$-axis intersects with the $c t$-axis and $c t_{\mathrm{A}}^{\prime}$-axis.

For example, among the lines which pass through $M_{1}$, the straight line parallel with the $x$-axis intersects the $c t_{\mathrm{A}}^{\prime}$-axis at point $\mathrm{A}_{4 / 5}$, and this is the point event of $\mathrm{W}_{\mathrm{A}}$ when $t=1(\mathrm{~s})$. Therefore $t_{\mathrm{A}}^{\prime}$ matches with eqn. (21).

The point events $\mathrm{A}_{4 / 5}$ and $\mathrm{B}_{0}$ are the point events of the two at the instant when $\mathrm{W}_{\mathrm{A}}$ and $\mathrm{W}_{\mathrm{B}}$ pass by each other. (Here, the point events $\mathrm{A}_{4 / 5}$ and $\mathrm{B}_{0}$ mean $t_{\mathrm{A}}^{\prime}=0.8(\mathrm{~s})$ and $\left.t_{\mathrm{B}}^{\prime}=0\right)$.

Also, the point events $\mathrm{M}_{2}$ and $\mathrm{B}_{4 / 5}$ are the point events of the two at the instant when $\mathrm{W}_{\mathrm{B}}$ passes in front of $\mathrm{W}$ of frame $S_{\mathrm{cl}}$ (Here, the point events $\mathrm{M}_{2}$ and $\mathrm{B}_{4 / 5}$ mean $t=2(\mathrm{~s})$ and $t_{\mathrm{B}}^{\prime}=0.8(\mathrm{~s})$ ).

\section{B. Triplet Thought Experiment 2 Performed by Observer M}

In this case, rocket $\mathrm{C}$ is taken to be the subject of consideration instead of rocket $\mathrm{B}$. In the first stage, just as in thought experiment 1 , 
Citation: Suto K (2018) The Problem of the Twin Paradox Elucidated Based on a Thought Experiment Carried out by Discriminating Between a Classically Stationary Frame and Moving Frame. J Phys Math 9: 278. doi: 10.4172/2090-0902.1000278

Page 6 of 8

observers $\mathrm{M}$ and $\mathrm{A}$ start their own stop watches $\mathrm{W}$ and $\mathrm{W}_{\mathrm{A}}$ when observer A passes in front of observer M (Figure 4a).

After that, when $0.8(\mathrm{~s})$ has elapsed on $\mathrm{W}$, rocket $\mathrm{C}$ passes in front of observer $\mathrm{M}$ at constant velocity $u$. When observer $\mathrm{C}$ on rocket $\mathrm{C}$ passes in front of observer $\mathrm{M}$, observer $\mathrm{M}$ stops $\mathrm{W}$, and observer $\mathrm{C}$ starts stop watch $\mathrm{W}_{\mathrm{C}}$ (Figure 6a).

Here, the velocity $u$ is the velocity at which rocket $\mathrm{C}$ approaches rocket $\mathrm{A}$ at a speed of $0.6 \mathrm{c}$.

To obtain the velocity of rocket C as seen from frame $S_{\mathrm{cl}}$, it is enough to substitute $0.6 \mathrm{c}$ for $v$ and $w$ in eqn. (17), and thus $u$ is:

$$
u=\frac{15}{17} c \text {. }
$$

Rocket $\mathrm{C}$ continues its motion at constant velocity, and when it has caught up with rocket $\mathrm{A}$, observer $\mathrm{A}$ stops $\mathrm{W}_{\mathrm{A}}$ and observer $\mathrm{C}$ stops $\mathrm{W}_{\mathrm{C}}$ (Figure 6b).

The situation of the thought experiment thus far can be explained with the following Minkowski diagram 2 (Figure 7).

The $c t^{\prime}$-axis of diagram 2 corresponds to the world line of stop watch $\mathrm{W}_{\mathrm{C}}$. In addition, the point events at the instant that $\mathrm{W}$ and $\mathrm{W}_{\mathrm{C}}$ pass by each other are $M_{4 / 5}$ and $C_{0}$. (Here, the point events $M_{4 / 5}$ and $C_{0}$ mean $t=0.8(\mathrm{~s})$ and $\left.t_{\mathrm{C}}^{\prime}=0\right)$.

Furthermore, the point events $\mathrm{A}_{2}$ and $\mathrm{C}_{4 / 5}$ are the point events of
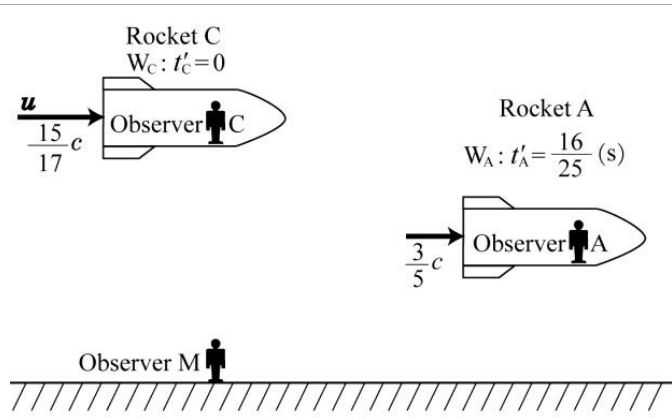

$$
\mathrm{W}: t=\frac{4}{5}(\mathrm{~s})
$$

Classically stationary frame

Figure 6a: Instant when $0.8(\mathrm{~s})$ has elapsed on $\mathrm{W}$ in the stationary frame, and $W_{C}$ of rocket $C$ passes in front of observer $M$.

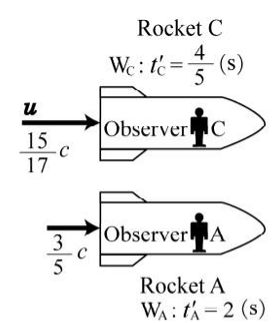

Observer M

11111111711111111111111111111111111111111111

$$
\mathrm{W}: t=\frac{4}{5}(\mathrm{~s})
$$

Classically stationary frame

Figure $6 \mathrm{~b}$ : When rocket $C$ has caught up with rocket $A$, observers $A$ and $C$ stop $\mathrm{W}_{\mathrm{A}}$ and $\mathrm{W}_{\mathrm{C}}$.

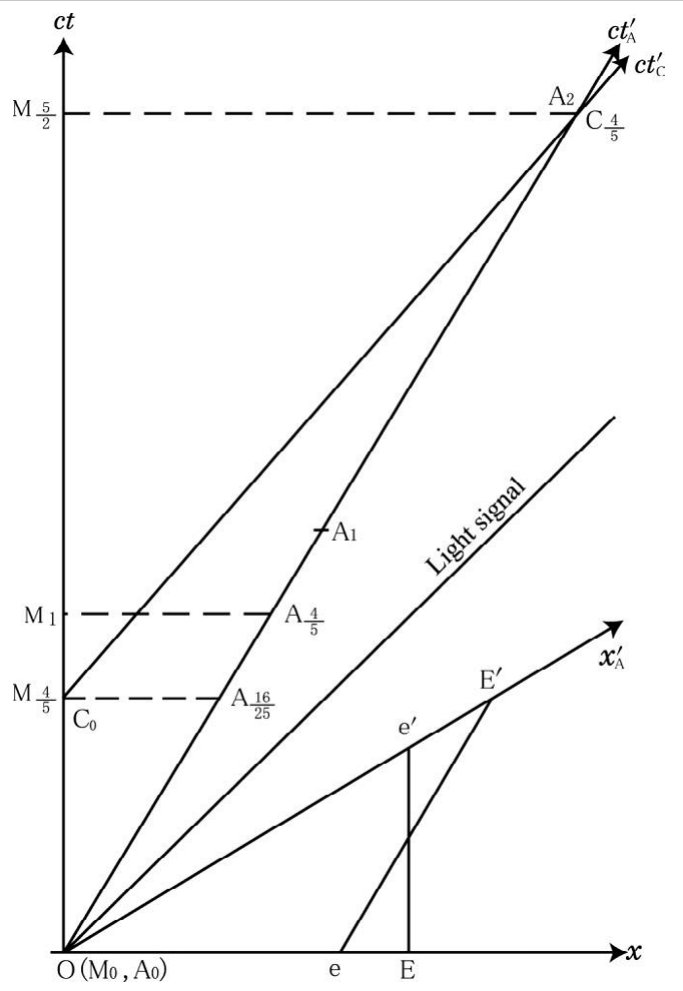

Figure 7: Minkowski diagram 2: This diagram corresponds to thought experiment 2. World lines of stop watches $W_{A}\left(A_{0} A_{2}\right), W_{C}\left(C_{0} C_{4 / 5}\right)$ and $\mathrm{W}\left(\mathrm{M}_{0} \mathrm{M}_{4 / 5}\right)$.

the two at the instant when $\mathrm{W}_{\mathrm{C}}$ has caught up with $\mathrm{W}_{\mathrm{A}}$. (Here, the point events $\mathrm{A}_{2}$ and $\mathrm{C}_{4 / 5}$ mean $t_{\mathrm{A}}^{\prime}=2(\mathrm{~s})$ and $t_{\mathrm{C}}^{\prime}=0.8(\mathrm{~s})$ ).

Also, in thought experiment 2, the observer in frame $S_{\mathrm{cl}}$, compares the time $t_{\mathrm{A}}^{\prime}$ elapsed on $\mathrm{W}_{\mathrm{A}}$ with the value $\left(t+t_{\mathrm{C}}^{\prime}\right)$ obtained by totalling the time $t$ elapsed on $\mathrm{W}$ with the time $t^{\prime}{ }_{\mathrm{c}}$ elapsed on $\mathrm{W}_{\mathrm{C}}$. Prior to that, the observer in frame $S_{\mathrm{cl}}$ compares $t$ with $t_{\mathrm{A}}^{\prime}$ and $t_{\mathrm{c}}^{\prime}$.

In order to find $t^{\prime}{ }_{\mathrm{A}}$ and $t_{\mathrm{c}}^{\prime}$, we first find $t_{\mathrm{A}}$ and $t_{\mathrm{c}}$ defined as follows. When $t_{\mathrm{A}}$ elapses on $\mathrm{W}, t_{\mathrm{A}}^{\prime}$ elapses on $\mathrm{W}_{\mathrm{A}}$, and when $t_{\mathrm{c}}$ elapses on $\mathrm{W}, t^{\prime}$. elapses on $\mathrm{W}_{\mathrm{C}}$. At this time, the following two equations hold.

$$
\begin{aligned}
& t_{\mathrm{A}}=\frac{4}{5}+t_{\mathrm{C}} . \\
& v t_{\mathrm{A}}=u t_{\mathrm{C}} .
\end{aligned}
$$

First, the following equation is obtained from eqns. (27) and (28).

$$
t_{\mathrm{A}}=\frac{4}{5} \frac{u}{u-v} .
$$

Here, if $0.6 \mathrm{c}$ is substituted for $v$, and the value of eqn. (26) is substituted for $u$,

$$
t_{\mathrm{A}}=2.5(\mathrm{~s}) \text {. }
$$

Here, $t_{\mathrm{A}}$ is the time elapsed on $\mathrm{W}$ during the $2(\mathrm{~s})$ when $\mathrm{W}_{\mathrm{A}}$ was operating.

Also, from eqns. (27) and (30),

$$
t_{\mathrm{c}}=1.7(\mathrm{~s})
$$

Here, $t_{\mathrm{c}}$ is the time elapsed on $\mathrm{W}$ during the interval when $\mathrm{W}_{\mathrm{C}}$ was operating. 
On the other hand, the time $t_{\mathrm{A}}^{\prime}$ elapsed in frame $S_{\mathrm{A}}^{\prime}$ is,

$t_{\mathrm{A}}^{\prime}=\frac{t_{\mathrm{A}}}{\gamma}=2(\mathrm{~s})$.

If $t_{\mathrm{C}}^{\prime}$ is taken to be the time which elapses on $\mathrm{W}_{\mathrm{C}}$ while $t_{\mathrm{c}}$ elapses on $\mathrm{W}$,

$t_{\mathrm{C}}^{\prime}=\frac{t_{\mathrm{C}}}{\gamma^{\prime}}, \gamma^{\prime}=\left(1-\frac{u^{2}}{c^{2}}\right)^{-1 / 2}$.

Here, when the value of eqn. (26) is substituted for $u$ in eqn. (33),

$\gamma^{\prime}=\frac{17}{8}$.

To find $t_{\mathrm{C}}^{\prime}$, it is sufficient to substitute the value of eqn. (31) for $t_{\mathrm{c}}$ in eqn. (33), and thus

$$
t_{\mathrm{C}}^{\prime}=\frac{4}{5}(\mathrm{~s})
$$

This $t_{\mathrm{C}}^{\prime}$ is the time over which $\mathrm{W}_{\mathrm{C}}$ was operating. Due to the above considerations, the observer $\mathrm{M}$ in frame $S_{\mathrm{cl}}$ obtains the following values for the elapsed times of $t_{\mathrm{A}}^{\prime} t$ and $t_{\mathrm{C}}^{\prime}$.

$$
\begin{aligned}
& t_{\mathrm{A}}^{\prime}=2(\mathrm{~s}), t=\frac{4}{5}(\mathrm{~s}), t_{\mathrm{C}}^{\prime}=\frac{4}{5}(\mathrm{~s}) . \\
& t_{\mathrm{A}}^{\prime}:\left(t+t_{\mathrm{C}}^{\prime}\right)=1: \frac{4}{5} .
\end{aligned}
$$

The value $\left(\begin{array}{ll}t & t^{\prime}\end{array}\right)$ obtained by totaling the times elapsed on $\mathrm{W}$ and $\mathrm{W}_{\mathrm{C}}$ is delayed compared to the time $t_{\mathrm{A}}^{\prime}$ which elapses in frame $S_{\mathrm{A}}^{\prime}$ which is not originally the stationary frame.

\section{Triplet Thought Experiment 3 Performed by Observer A on} Rocket A

According to Einstein's "principle of relativity," the two inertial frames are equivalent, and thus the same results are obtained no matter which inertial frame measurement is carried out from. The coordinate system of rocket A is not a classically stationary frame, but relativistically it is a stationary frame.

The observer in rocket A regards his own coordinate system as a stationary frame. Therefore, eqns. (36) and (37) are interpreted as follows. (note the change in the dash' indicating the moving frame due to the change in the stationary frame).

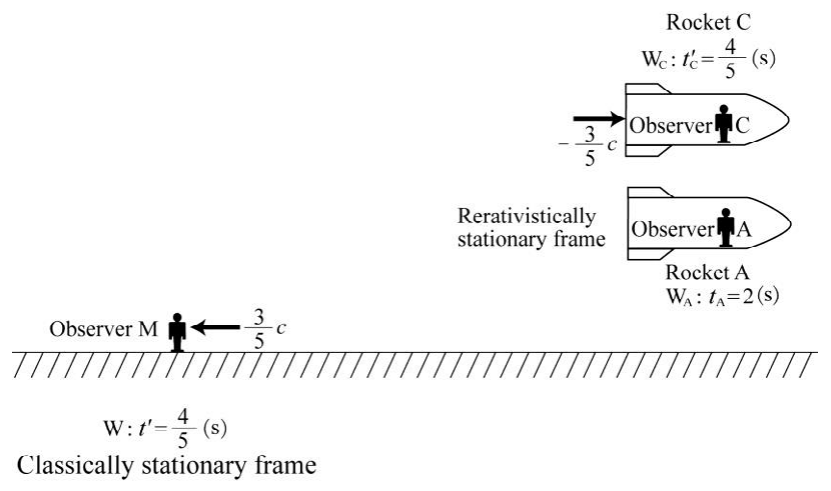

Figure 8: Thought experiment 3 can be interpreted as indicating that observer A has carried out thought experiment 1, taking frame $S_{\mathrm{A}}^{\prime}$ as the stationary frame.

$$
\begin{aligned}
& t_{\mathrm{A}}=2(\mathrm{~s}), t^{\prime}=\frac{4}{5}(\mathrm{~s}), t_{\mathrm{C}}^{\prime}=\frac{4}{5}(\mathrm{~s}) . \\
& t_{\mathrm{A}}:\left(t^{\prime}+t_{\mathrm{C}}^{\prime}\right)=1: \frac{4}{5} .
\end{aligned}
$$

Eqn. (39) can be interpreted as indicating that observer A has conducted the triplet thought experiment. (Figure 8) Eqns. (24) and (39) are the experiment results predicted by the STR.

\section{Discussion}

Let us consider, as far as possible, the a priori rhythm by which the stop watches used in thought experiments mark off time. The problem of rhythm cannot be addressed in the STR, but this paper has introduced $S_{\mathrm{cl}}$ and thus it is possible to discuss the problem of rhythm.

Now, if the rhythms by which the three stop watches mark time are expressed as rhythm $(\mathrm{W})$, rhythm $\left(\mathrm{W}_{\mathrm{A}}\right)$ and rhythm $\left(\mathrm{W}_{\mathrm{B}}\right)$, then observer $\mathrm{M}$ can predict the following relationship from eqn. (23).

$$
\text { rhythm }(\mathrm{W}) \text { : rhythm }\left(\mathrm{W}_{\mathrm{A}}\right): \text { rhythm }\left(\mathrm{W}_{\mathrm{B}}\right)=1: \frac{4}{5}: \frac{4}{5} \text {. }
$$

Also, in thought experiment 2, observer $\mathrm{M}$ can predict the following relationship if eqn. (34) is taken into account.

$$
\text { rhythm }\left(\mathrm{W}_{\mathrm{A}}\right) \text { : rhythm }(\mathrm{W}) \text { : rhythm }\left(\mathrm{W}_{\mathrm{C}}\right)=\frac{4}{5}: 1: \frac{8}{17} \text {. }
$$

In contrast, the observer in rocket $\mathrm{A}$ regards his own coordinate frame as a stationary frame, and interpreted the situation as in eqn. (38). However, the problem of rhythm cannot be addressed with the STR, and thus the observer in rocket A cannot make the following prediction from eqn (38) [9-12]. That is,

rhythm $\left(\mathrm{W}_{\mathrm{A}}\right)$ : rhythm $(\mathrm{W})$ : rhythm $\left(\mathrm{W}_{\mathrm{C}}\right) \neq 1: \frac{4}{5}: \frac{4}{5}$.

Rhythm is an a priori concept, and thus it is not possible for all observers to assert that there will be delay in the rhythm of a clock in an inertial frame moving at constant velocity relative to their own inertial frame.

Rocket A undergoes accelerated motion until it attains motion at constant velocity. The coordinate system of rocket A is clearly a moving frame. The two inertial frames $\mathrm{M}$ and $\mathrm{A}$ are by no means equivalent. Even so, the author was able to confirm the delay in time predicted by the STR even in the triplet thought experiment carried out by observer A.

\section{Conclusion}

It is evident that the following relationships hold in the STR.

$$
\begin{aligned}
& t=2(\mathrm{~s}), t_{\mathrm{A}}^{\prime}=\frac{4}{5}(\mathrm{~s}), t_{\mathrm{B}}^{\prime}=\frac{4}{5}(\mathrm{~s}) . \\
& t_{\mathrm{A}}=2(\mathrm{~s}), t^{\prime}=\frac{4}{5}(\mathrm{~s}), t_{\mathrm{C}}^{\prime}=\frac{4}{5}(\mathrm{~s}) .
\end{aligned}
$$

However, even if eqns. (43) and (44) hold, it is not the case that the following relationships hold.

Rhythm predicted by observer M:

$$
\text { rhythm }(\mathrm{W}): \text { rhythm }\left(\mathrm{W}_{\mathrm{A}}\right)=1: \frac{4}{5} \text {. }
$$

Rhythm predicted by observer A: 
Citation: Suto K (2018) The Problem of the Twin Paradox Elucidated Based on a Thought Experiment Carried out by Discriminating Between a Classically Stationary Frame and Moving Frame. J Phys Math 9: 278. doi: 10.4172/2090-0902.1000278

Page 8 of 8

rhythm $\left(\mathrm{W}_{\mathrm{A}}\right)$ : rhythm $(\mathrm{W})=1: \frac{4}{5}$.

In the STR, the discussion never extends to the problem of rhythm, which is an a priori concept. Therefore, in the STR no contradictions arise due to discussion of rhythm.

In this paper, the following relationships were derived by incorporating frame $S_{\mathrm{Cl}}$, into the thought experiment.

$$
\begin{aligned}
& t=2(\mathrm{~s}), t_{\mathrm{A}}^{\prime}=\frac{4}{5}(\mathrm{~s}), t_{\mathrm{B}}^{\prime}=\frac{4}{5}(\mathrm{~s}) . \\
& t=\frac{4}{5}(\mathrm{~s}), t_{\mathrm{A}}^{\prime}=2(\mathrm{~s}), t_{\mathrm{C}}^{\prime}=\frac{4}{5}(\mathrm{~s}) .
\end{aligned}
$$

The a priori rhythms with which the three clocks (stop watches) mark time are as follows.

$$
\begin{aligned}
& \text { rhythm }(\mathrm{W}): \operatorname{rhythm}\left(\mathrm{W}_{\mathrm{A}}\right): \operatorname{rhythm}\left(\mathrm{W}_{\mathrm{B}}\right)=1: \frac{4}{5}: \frac{4}{5} \text {. } \\
& \text { rhythm }(\mathrm{W}): \operatorname{rhythm}\left(\mathrm{W}_{\mathrm{A}}\right): \operatorname{rhythm}\left(\mathrm{W}_{\mathrm{C}}\right)=1: \frac{4}{5}: \frac{8}{17}
\end{aligned}
$$

However, in the STR all inertial frames are regarded as equivalent, and thus in thought experiment 3 , the coordinate system of rocket $\mathrm{A}$ becomes a stationary frame. Thus, the observer in rocket $\mathrm{A}$ interprets eqn. (48) as eqn. (44). (Here, $t_{\mathrm{A}}^{\prime}$ has been changed to $t_{\mathrm{A}}$, and $t$ to $t^{\prime}$ ).

However, it is possible to conclude eqn. (44) because eqn. (48) holds, and eqn. (48) holds because eqn. (50) holds. Even if eqn. (44) is assumed to hold, it is not the case that the following relationships hold.

rhythm $\left(\mathrm{W}_{\mathrm{A}}\right)$ : rhythm $(\mathrm{W})$ : rhythm $\left(\mathrm{W}_{\mathrm{C}}\right)=1: \frac{4}{5}: \frac{4}{5}$.

The correct relationships are the following.

$$
\text { rhythm }\left(\mathrm{W}_{\mathrm{A}}\right) \text { : rhythm }(\mathrm{W}) \text { : rhythm }\left(\mathrm{W}_{\mathrm{C}}\right)=1: \frac{5}{4}: \frac{10}{17} \text {. }
$$

If the mechanism of these relationships can be understood, the twin paradox will no longer be a paradox. The STR was constructed without taking into account the existence of velocity vectors existing in the natural world, and thus became a theory containing a contradiction.

\section{Acknowledgments}

I would like to express my thanks to the staff at ACN Translation Services for their translation assistance. Also, I wish to express my gratitude to $\mathrm{Mr}$. $\mathrm{H}$. Shimada for drawing figures.

\section{References}

1. French A P (1968) Special Relativity. Norton\&Company 154-159.

2. Holton G (1963) Selected Reprints in Special Relativity Theory. American Institute of Physics, New York.

3. Einstein A (1923) The Principle of Relativity. New York, Dover, USA, p. 41.

4. Suto K (2010) Violation of the Special Theory of Relativity as Proven by Synchronization of Clocks. Physics Essays 23: 511.

5. Suto K (2014) Velocity Addition Laws which can Coexist with the Velocity Addition Law in the Special Theory of Relativity. Physics Essays 27: 191.

6. Suto K (2015) Demonstration of the Existence of a Velocity Vector Missing from the Special Theory of Relativity. Physics Essays 28: 345.

7. Suto K (2017) Method of Verifying the Existence of Velocity Vectors Excluded by the Special Theory of Relativity. J Phys Math 4: 258.

8. Suto K (2014) True Factors Determining the Ratio of Space Contraction and Time Dilation Predicted by the Special Theory of Relativity. Physics Essays 27: 580 .

9. Suto K (2016) Thought Experiment Revealing a Contradiction in the Special Theory of Relativity. Applied Physics Research 8: 70

10. Suto K (2017) An Elucidation of the Symmetry of Length Contraction Predicted by the Special Theory of Relativity. Applied Physics Research 9: 31.

11. Suto K (2016) Elucidation of Time Symmetry Predicted by the Special Theory of Relativity. IOSR J Appl Phys 8: 20.

12. Suto K (2017) A New Problem with the Twin Paradox. Applied Physics Research 9: 77. 